\title{
PROSPECTS FOR DETERMINING THE EQUATION OF STATE OF THE DARK ENERGY: WHAT CAN BE LEARNED FROM MULTIPLE OBSERVABLES?
}

\author{
Jens Kujat, ${ }^{1}$ Angela M. Linn, ${ }^{1}$ Robert J. Scherrer, ${ }^{1,2}$ and David H. Weinberg ${ }^{2}$ \\ Received 2001 December 11; accepted 2002 February 13
}

\begin{abstract}
The dark energy that appears to produce the accelerating expansion of the universe can be characterized by an equation of state $p=w \rho$ with $w<-\frac{1}{3}$. A number of observational tests have been proposed to study the value or redshift dependence of $w$, including Type Ia supernova distances, the Sunyaev-Zeldovich effect, cluster abundances, strong and weak gravitational lensing, galaxy and quasar clustering, galaxy ages, the Ly $\alpha$ forest, and cosmic microwave background anisotropies. The proposed observational tests based on these phenomena measure either the distance-redshift relation $d(z)$, the Hubble parameter $H(z)$, the age of the universe $t(z)$, the linear growth factor $D_{1}(z)$, or some combination of these quantities. We compute the evolution of these four observables and of the combination $H(z) d(z)$ that enters the Alcock-Paczyznski anisotropy test in models with constant $w$, in quintessence models with some simple forms of the potential $V(\phi)$, and in toy models that allow more radical time variations of $w$. Measurement of any of these quantities to a precision of a few percent is generally sufficient to discriminate between $w=-1$ and $-\frac{2}{3}$. However, the time dependence predicted in quintessence models is extremely difficult to discern because the quintessence component is dynamically unimportant at the redshifts where $w$ departs substantially from its low- $z$ value. Even for the toy models that allow substantial changes in $w$ at low redshift, there is always a constant- $w$ model that produces very similar evolution of all of the observables simultaneously. We conclude that measurement of the effective equation of state of the dark energy may be achieved by several independent routes in the next few years but that detecting time variation in this equation of state will prove very difficult except in specialized cases.
\end{abstract}

Subject headings: cosmological parameters — cosmology: theory

\section{INTRODUCTION}

The big cosmological surprise of recent years is that the dominant form of energy in the universe has negative pressure and is therefore causing the expansion of the universe to accelerate. The most direct evidence for acceleration comes from the Hubble diagram of Type Ia supernovae (SNe Ia), in particular, the relative apparent brightness of SNe Ia at redshifts $z \sim 0$ and $z \sim 0.5-1$ (Riess et al. 1998; Perlmutter et al. 1999). However, other strong arguments for a "dark energy" component follow from combining the cosmic microwave background (CMB) evidence for a spatially flat universe (Netterfield et al. 2002; Pryke et al. 2002) with either a minimum age $t_{0} \sim 13$ Gyr (Vandenberg, Stetson, \& Bolte 1996) or dynamical evidence that the density of clustered matter is well below the critical density (see Bahcall, Fan, \& Cen 1997, Carlberg, Yee, \& Ellingson 1997, and Weinberg et al. 1999b for examples of three distinct routes to this conclusion, although there are many others). The first combination, together with a Hubble constant $H_{0} \approx 70 \mathrm{~km} \mathrm{~s}^{-1} \mathrm{Mpc}^{-1}=(14 \mathrm{Gyr})^{-1}$ (Freedman et al. 2001), requires a component whose gravitational acceleration roughly cancels the gravitational deceleration caused by the pressureless matter so that $t_{0} \approx H_{0}^{-1}$. The second combination requires that the dominant form of energy be unclustered, although it implies nothing more specific about its equation of state. A more model-dependent argument

\footnotetext{
${ }^{1}$ Department of Physics, Ohio State University, 174 West 18th Avenue, Columbus, OH 43210

${ }^{2}$ Department of Astronomy, Ohio State University, 140 West 18 th Avenue, Columbus, OH 43210.
}

for a negative pressure component comes from the success of inflationary models with cold dark matter (CDM) and a cosmological constant $(\Lambda)$ in matching a variety of constraints from CMB anisotropies and large-scale structure measurements (see Wang, Tegmark, \& Zaldarriaga 2001 for a recent review).

In this paper, we explore the prospects for determining the equation of state of the dark energy component through a variety of observational methods. A true cosmological constant can be treated as a vacuum energy with timeindependent density and pressure related by $p=-\rho$. Current observations favor an equation of state fairly close to this prediction (Garnavich et al. 1998). However, a number of authors have considered the more general possibility that the negative pressure component is a scalar field (aka "quintessence") with energy density determined by its potential and effective equation of state $p=w \rho$, where $w$ can be constant or time-varying (Ratra \& Peebles 1988; Turner \& White 1997; Caldwell, Dave, \& Steinhardt 1998). Interest in models with time-varying $w$ has been spurred by arguments that certain simple potentials lead "naturally" to a negative pressure quintessence component that dominates the expansion at late times, independent of the initial conditions (Zlatev, Wang, \& Steinhardt 1999; Steinhardt, Wang, \& Zlatev 1999). Variants on this theme include fields with a nonstandard kinetic term (Armendariz-Picon, Mukhanov, \& Steinhardt 2000) or models with a complex scalar field (Boyle, Caldwell, \& Kamionkowski 2001).

Further afield, there is the possibility that the negative pressure component is a network of frustrated topological defects (Vilenkin 1985; Spergel \& Pen 1997) or that cosmic acceleration arises from a breakdown of general relativity rather than the addition of a new energy component 
(Mannheim 2001; see also Tegmark 2001). The hope, thus far unrealized, is that one of these ideas will eventually provide a natural explanation of why the vacuum energy density is 120 orders of magnitude below the Planck scale and why it is comparable to the matter density at the present day without having to resort to anthropic selection arguments (Efstathiou 1995; Martel, Shapiro, \& Weinberg 1998).

Any clear evidence that $w \neq-1$ or, better still, that $w$ varies in time would provide crucial clues toward understanding the physics of the dark energy. Through its influence on the cosmic expansion history, this component affects many observable phenomena, including CMB anisotropies, the Ly $\alpha$ forest, strong and weak gravitational lensing, the anisotropy of quasar and galaxy clustering in redshift space, the ages of the oldest galaxies as a function of redshift, and standard-candle or standard-ruler measurements of the distance-redshift relation. This paper discusses these potential observational tests in a unified fashion. The equation of state determines the history of the energy density $\rho_{\phi}$, which together with the densities $\rho_{m}$ and $\rho_{r}$ of matter and radiation, respectively, determines the evolution of the Hubble parameter $H(z)$ via the Friedmann equation. The history of $H(z)$ in turn determines the age of the universe $t(z)$, the growth factor of linear perturbations $D_{1}(z)$, and distance measures like the angular diameter distance $d_{A}(z)$ or luminosity distance $d_{L}(z)$, which are related to each other by cosmology-independent powers of $(1+z)$. Essentially all proposed tests of the properties of the negative pressure component amount to measurements of $H(z), t(z), D_{1}(z)$, or $d(z)$, or some combination of them, at redshifts accessible to a particular observational technique. We will investigate the dependence of these four quantities and of the specific combination $H(z) d_{A}(z)$ that is constrained by the AlcockPaczynski (1979, hereafter AP) anisotropy test on the value and time history of $w$.

Our paper joins and, we hope, complements a flood of recent papers that examine the prospects for specific tests and specific data sets in much greater detail. Since the strongest evidence for $\Lambda$ or a quintessence component comes from SN Ia observations, and substantial improvements are likely from ground-based campaigns and possibly a dedicated satellite (the Supernova/Acceleration Probe $[S N A P]^{3}$ ), many authors have examined the extent to which present or future SN Ia observations can constrain $w(z)$ (Turner \& White 1997; Garnavich et al. 1998; Astier 2001; Chiba \& Nakamura 2000; Huterer \& Turner 2001; Saini et al. 2000; Barger \& Marfatia 2001; Chevallier \& Polarski 2001; Maor, Brustein, \& Steinhardt 2001; Ng \& Wiltshire 2001; Podariu, Nugent, \& Ratra 2001; Wang \& Garnavich 2001; Wang \& Lovelace 2001; Weller \& Albrecht 2001). Because CMB anisotropy predictions depend most strongly on the sum of $\rho_{\phi}$ and $\rho_{m}$, while SN Ia distances depend more nearly on the difference, the combination of these complementary observations yields much tighter constraints on the negative pressure component than either does alone (Caldwell et al. 1998; Efstathiou 1999; Baccigalupi et al. 2002; Corasiniti \& Copeland 2002; Doran, Lilley, \& Wetterich 2002). The SunyaevZeldovich effect or size of radio sources offer alternative ways of measuring $d_{A}(z)$ (Birkinshaw 1999; Lima \& Alcaniz 2002 ), and the volume-redshift test using galaxy counts constrains the combination $d_{A}^{2}(z) H^{-1}(z)$ (Newman \& Davis

\footnotetext{
${ }^{3}$ See the SNAP Web site at http://snap.lbl.gov.
}

$2000,2002)$. The evolution of the galaxy cluster mass function can constrain the linear growth factor $D_{1}(z)$ (Benabed \& Bernardeau 2001; Doran, Schwindt, \& Wetterich 2001; Haiman, Mohr, \& Holder 2001; Newman et al. 2002; Weller, Battye, \& Kneissl 2001), and population synthesis modeling of galaxy spectra can constrain $t(z)$ (Lima \& Alcaniz 2000). Jimenez \& Loeb (2001) suggest that relative galaxy ages can be used to measure $d z / d t$ and, thus, $H(z)$. Hui (1999) and Huterer (2002) have examined constraints on $w$ that can be obtained from weak lensing, while $\mathrm{Hu}$ (2002) has considered lensing in combination with the CMB. Calvão, de Mello Neto, \& Waga (2002) have discussed constraints that could be obtained by applying the AP test to the $2 \mathrm{dF}$ quasar redshift survey of Boyle et al. (2000; for related discussions, see Hui, Stebbins, \& Burles 1999; Cappi 2002; Dalal et al. 2001; McDonald 2001).

Most of these papers have considered the potential observational constraints singly or in pairs. The goals of our more abstract discussion, in which we consider all of these observables together but do not focus on specific observational strategies, are twofold. First, we aim to understand what level of precision is necessary with any of these quantities to obtain useful constraints on $w$. Second, we want to know whether these different observables provide complementary information about the time variation of $w$, breaking degeneracies that exist for a single measure by probing different aspects of the expansion history. Unfortunately, our conclusions on the latter point are pessimistic - there are many different ways to measure $w$, but distinguishing a time-varying $w$ from a constant $w$ is likely to prove difficult. The papers by Wang et al. (2000) and Tegmark (2001) also consider multiple observables, focusing on present constraints and future prospects, respectively. Tegmark's paper, in particular, is similar in spirit to ours but different in the way that it frames the problem and evaluates the prospects.

In the next section we discuss the various quintessence models that we examine in this paper. We discuss the observables in $\S 3$, beginning with the formulas that relate these quantities to the expansion history and proceeding to a brief account of observations that might measure these quantities in the next few years. We present our results in $\S 4$, first for the quintessence models described in $\S 2$, then for a class of "toy" models designed to allow stronger time variation of $w$ at low redshift. We summarize our conclusions in $\S 5$.

\section{QUINTESSENCE MODELS}

We will adopt the language and calculational framework of quintessence models, although most of our general conclusions are also relevant to other possible explanations of cosmic acceleration, such as those mentioned in $\S 1$. Furthermore, in light of evidence from the location of the first acoustic peak in the CMB anisotropy spectrum (Netterfield et al. 2002; Pryke et al. 2002), we will restrict our attention to spatially flat models.

The Friedmann equation for a spatially flat, expanding universe can be written

$$
\frac{\dot{a}}{a} \equiv H(z)=H_{0} \sqrt{\sum_{i} \Omega_{i, 0} \frac{\rho_{i}(z)}{\rho_{i, 0}}} .
$$

Here $a$ is the scale factor, $\dot{a}$ is the derivative of the scale 
factor with respect to time $t, H_{0}$ is the value of the Hubble parameter at the present time $t_{0}$, and $\Omega_{i, 0}$ is the present density of some $i$ th component of the energy density relative to the present critical density $\left(\Omega_{i, 0} \equiv \rho_{i, 0} / \rho_{c, 0}\right)$. For adiabatic expansion, the energy density of a component with equation of state $p_{i}=w_{i} \rho_{i}$ with constant $w_{i}$ evolves with redshift as

$$
\begin{aligned}
\frac{\rho_{i}(z)}{\rho_{i, 0}} & =(1+z)^{n_{i}}, \\
n_{i} & \equiv 3\left(1+w_{i}\right) .
\end{aligned}
$$

Normal matter has $w_{i}=0$ and $n_{i}=3$, while radiation has $w_{i}=\frac{1}{3}$ and $n_{i}=4$. A true cosmological constant, with $\rho_{i}=$ const, $n_{i}=0$, has $w_{i}=-1$. We will often refer to models in terms of the energy density scaling index $n$, defined by equation (2), rather than by $w$ itself since the value of $n$ more directly captures the impact of a component on the expansion history.

A coasting expansion, in which comoving observers have constant velocity, has $H(z) \propto(1+z)$. An accelerated expansion requires, at a minimum, that the dominant energy component have $n_{i}<2$ and, thus, $w_{i}<-\frac{1}{3}$. (More precisely, $\langle w\rangle$, the density-weighted average value of $w$, must satisfy $\langle w\rangle<-\frac{1}{3}$.) Quintessence, a term reintroduced to cosmology by Caldwell et al. (1998) after millennia of neglect, refers generically to a scalar field with equation of state $p_{\phi}=w_{\phi} \rho_{\phi}$ and $w_{\phi}<0$. The first class of models that we consider are those in which $w_{\phi}$ is constant. In this case, the Friedmann equation can be written

$$
\begin{aligned}
H(z) & \equiv \frac{\dot{a}}{a} \\
& =H_{0} \sqrt{\Omega_{r, 0}(1+z)^{4}+\Omega_{m, 0}(1+z)^{3}+\Omega_{\phi, 0}(1+z)^{n}},
\end{aligned}
$$

with $n$ given by equation (3).

More general models often treat quintessence as a minimally coupled scalar field $\phi$, obeying the equation

$$
\ddot{\phi}=-3 H \dot{\phi}-\frac{d V}{d \phi},
$$

where $w$ for the scalar field is

$$
w=\frac{(1 / 2) \dot{\phi}^{2}-V(\phi)}{(1 / 2) \dot{\phi}^{2}+V(\phi)} .
$$

When $V(\phi)$ is an exponential or a negative power law, the scalar field has the desirable property that its final evolution is independent of initial conditions, a behavior that has been dubbed "tracking" (Zlatev et al. 1999; Steinhardt et al. 1999). The negative power-law potentials lead to constant $w$ when the contribution from the scalar field energy density is subdominant (Ratra \& Peebles 1988; Liddle \& Scherrer 1999), but when the scalar field energy density comes to dominate at late times, the value of $w$ changes. In principle, then, such models should be observationally distinguishable from models with constant $w$.

For our second class of models, we have chosen a subset of the negative power-law potentials, where

$$
V(\phi) \propto \phi^{\alpha},
$$

with $\alpha<0$. If the dominant component has a density that

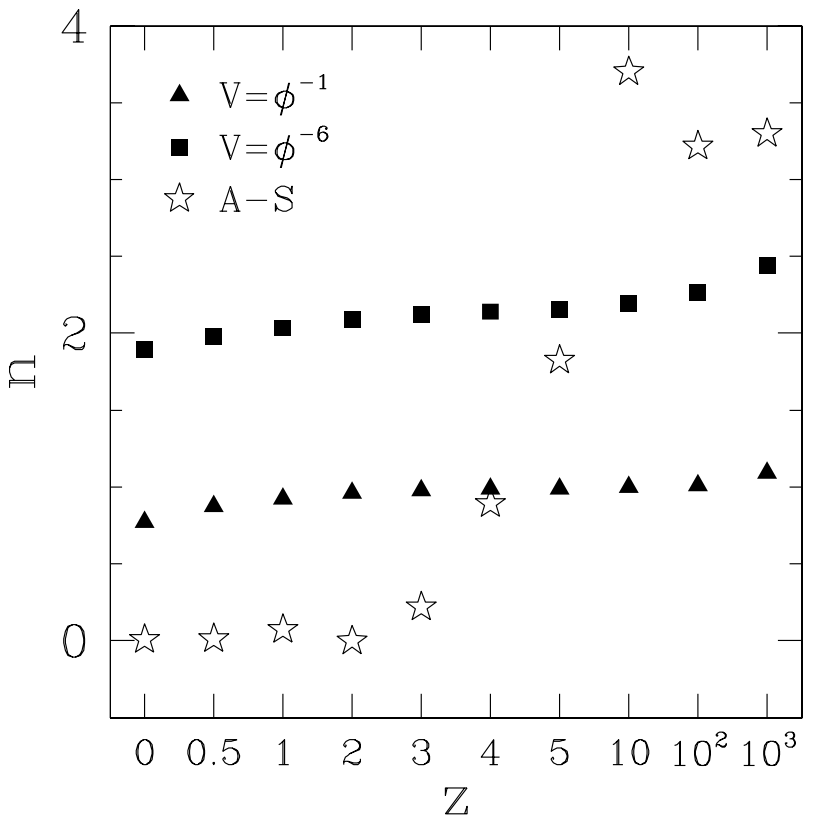

FIG. 1. - Evolution of $n \equiv d \log \rho_{\phi} / d \log (1+z)$ as a function of redshift $z$ for a scalar field with the indicated potential and a cosmological model with $\Omega_{m, 0}=0.4, \Omega_{r, 0}=9.8 \times 10^{-5}$, and $\Omega_{\phi, 0}=1-\Omega_{m, 0}-\Omega_{r, 0} \approx 0.6$. In this figure, and in all other figures in the paper, the horizontal axis effectively represents a set of discrete bins, so it is neither linear nor logarithmic.

scales as $\rho \propto(1+z)^{m}$ (e.g., $m=4$ during the radiationdominated era and $m=3$ during the matter-dominated era), then these models have

$$
n=\left[\frac{\alpha}{(\alpha-2)}\right] m
$$

when $\rho_{\phi} \ll \rho_{m}$ (Liddle \& Scherrer 1999). At late times, when the scalar field energy density begins to dominate, equation (8) no longer holds, and $n$ changes with time. We have chosen to examine two representative cases: $\alpha=-1$ and $\alpha=-6$. This choice is somewhat arbitrary, but these are the same cases that are discussed by Zlatev et al. (1999).

The evolution of $n$ in these models is displayed in Figure 1, assuming cosmological parameter values $\Omega_{m, 0}=0.4, \Omega_{r, 0}=9.8 \times 10^{-5}$, and $\Omega_{\phi, 0}=1-\Omega_{m, 0}-\Omega_{r, 0}$. (This value of $\Omega_{r, 0}$ corresponds to a photon temperature of $T=2.73 \mathrm{~K}$, a standard neutrino population, and a Hubble parameter of $H_{0}=65 \mathrm{~km} \mathrm{~s}^{-1} \mathrm{Mpc}^{-1}$. This is the only place in our calculations where $H_{0}$ enters, and it has a very small effect on our results.) For these models, we define $n(z)$ to be the local logarithmic derivative of $\rho_{\phi}$ with respect to $(1+z)$. Figure 1 shows that the value of $n$ for $3 \lesssim z \lesssim 10$ is almost exactly constant and given by equation (8), namely, $n=1$ for $\alpha=-1$ and $n=9 / 4$ for $\alpha=-6$. At $z<3, n$ decreases slightly, reaching present-day values of $n=0.77$ for $\alpha=-1$ and $n=1.89$ for $\alpha=-6$. In $\S 4$ we will see whether cosmological tests can detect these slight changes in $n$.

Although models with an exponential potential are quite natural and can lead to $\rho_{\phi} \approx \rho_{m}$ at all times, they are ruled out for several reasons: they tend to give values of $\rho_{\phi}$ too large during primordial nucleosynthesis (Ferreira \& Joyce 1997), and they lead to $n=3$ at late times, which does not produce an accelerated expansion. These problems are remedied in the model of Albrecht \& Skordis (2000), who intro- 


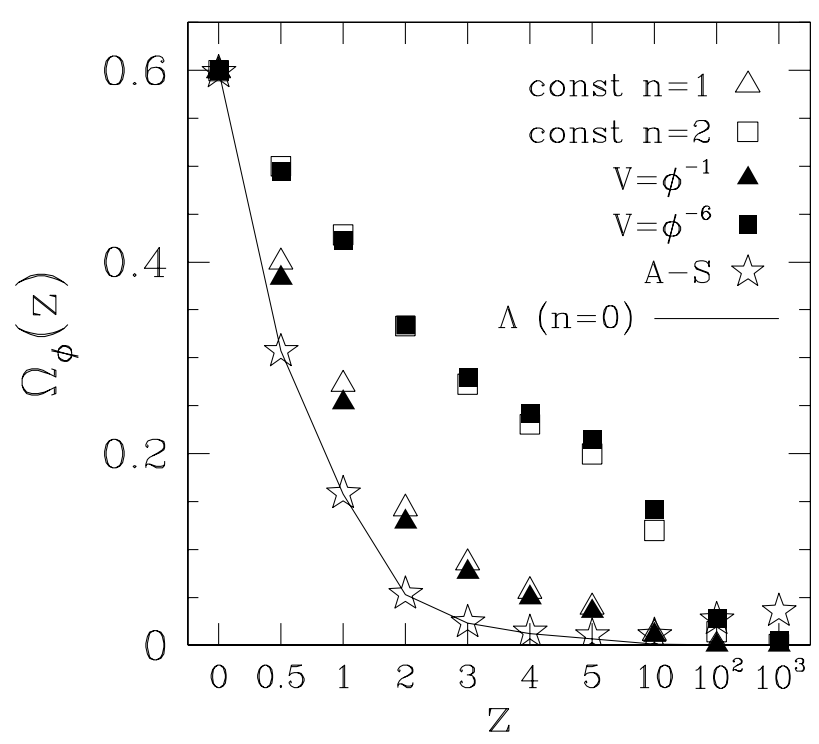

FIG. 2. $-\Omega_{\phi}$ as a function of redshift $z$ for the indicated quintessence models. The solid curve is $\Omega_{\Lambda}(z)$ for a cosmological constant.

duced a potential consisting of an exponential multiplied by a polynomial,

$$
V(\phi)=\left[(\phi-B)^{\gamma}+A\right] e^{-\lambda \phi},
$$

where $A, B, \gamma$, and $\lambda$ are constants. These constants can be chosen to produce a model for which $n=m$ at early times, when the scalar field is sliding down the exponential potential, but $n=0$ at late times, when the scalar field settles into the local minimum in the potential. The constants in this model must still be tuned to give the desired value for $\Omega_{\phi, 0}$; following Albrecht \& Skordis (2000), we have examined a model with $A=0.01, B=34.8, \gamma=2$, and $\lambda=8$ and with initial conditions chosen so as to fix $\Omega_{m, 0}=0.4$ today. The evolution of $n$ for this model is also shown in Figure 1. It exhibits a sharp transition from $n \approx 3.5$ at $z \geq 10$ to $n \approx 0$ at $z<3$, a much more dramatic change than that in the power-law scalar field models. (Exponential potentials can also be made to work in models in which the scalar field is coupled to matter [Amendola 2000]; however, we confine our attention in this paper to minimally coupled fields.)

The dynamical significance of the quintessence component is quantified by the density parameter $\Omega_{\phi}(z)$. Figure 2 shows the evolution of $\Omega_{\phi}(z)$ for a cosmological constant (solid curve) and the five quintessence models discussed above. The $n=0$ (cosmological constant), $n=1$, and $n=2$ cases are quite distinct, as one would expect from their differing values of $\rho_{\phi}(z)$. However, the $V(\phi)=\phi^{-1}$ case closely parallels the constant $n=1$ case, and the $V(\phi)=\phi^{-6}$ case likewise tracks the model with constant $n=2$. The Albrecht-Skordis $\Omega_{\phi}$ is nearly indistinguishable from that of a cosmological constant except at high redshift, where the change in $n$ makes a small but noticeable difference.

\section{THE OBSERVABLES}

Our starting point is the Friedmann equation in the form of equation (1). As components we consider matter with $\Omega_{m, 0}=0.4$, radiation with $\Omega_{r, 0}=9.8 \times 10^{-5}$, and quintessence with $\Omega_{\phi, 0}=1-\Omega_{m, 0}-\Omega_{r, 0} \approx 0.6$. We compute the ratio $\rho_{\phi}(z) / \rho_{\phi, 0}$ from equation (2) for constant- $w$ models [thus obtaining eq. (4) for $H(z)$ ] or by computing the evolution of $\phi$ from the dynamical equation (5) for the negative power law or Albrecht-Skordis models.

The Friedmann equation directly determines the behavior of our first observable, the Hubble parameter $H(z)$. We compute other observables given $H(z)$ via the standard treatments in, e.g., Peebles (1980, 1993), Kolb \& Turner (1990), or Hogg (1999). The age of the universe at redshift $z$ is

$$
t(z)=\int_{z}^{\infty} \frac{d z^{\prime}}{\left(1+z^{\prime}\right) H\left(z^{\prime}\right)} .
$$

The angular diameter distance $d_{A}(z)$, which is the ratio of the comoving size of an object to its angular size in radians, is

$$
d_{A}(z)=\frac{c}{(1+z)} \int_{0}^{z} \frac{d z^{\prime}}{H\left(z^{\prime}\right)} .
$$

Other distances, e.g., those that affect the SN Ia Hubble diagram or gravitational lensing predictions, are related to $d_{A}$ by powers of $(1+z)$; the bolometric luminosity distance, for example, is $d_{L}(z)=(1+z)^{2} d_{A}(z)$. Since these factors are independent of the cosmological model, a measurement of any of these distances determines all of them to the same fractional accuracy, so we take $d_{A}(z)$ as our representative observable for all distance measures.

The linear growth factor $D_{1}$ is defined by the relation

$$
\delta^{(1)}(\boldsymbol{x}, t)=\delta(\boldsymbol{x}) D_{1}(t),
$$

where $\delta^{(1)}(\boldsymbol{x}, t)$ is the first-order density perturbation. We choose the normalization $D_{1}(z=0)=1$, so that $D_{1}(z)=$ $\delta^{(1)}(z) / \delta^{(1)}(0)$ gives the linear growth of perturbations between redshift $z$ and redshift 0 . Then $D_{1}$ is the growingmode solution to the differential equation

$$
\ddot{D}_{1}+2 H(z) \dot{D}_{1}-\frac{3}{2} \Omega_{m, 0} H_{0}^{2}(1+z)^{3} D_{1}=0 .
$$

For fixed $\Omega_{m, 0}, D_{1}(z)$ is a function only of $H(z)$, so it is again determined by the Friedmann equation. We solve this equation for $D_{1}(z)$ with a standard Runge-Kutta integration method. In the pure cosmological constant case, a closed form expression for $D_{1}(z)$ is

$$
D_{1}(z)=\frac{H(z)}{H_{0}} \int_{z}^{\infty} \frac{d z^{\prime}\left(1+z^{\prime}\right)}{H^{3}\left(z^{\prime}\right)}\left[\int_{0}^{\infty} \frac{d z^{\prime}\left(1+z^{\prime}\right)}{H^{3}\left(z^{\prime}\right)}\right]^{-1},
$$

where the factor in brackets enforces our normalization definition (Eisenstein 1997, based on Heath 1977). Unfortunately, this expression generalizes only to the case $n=2$, but it does illustrate that the linear growth factor weights the expansion history in a different way than does the age or distance. For constant $w$, a solution for $D_{1}(z)$ can be found in terms of hypergeometric functions (Silveira \& Waga 1994).

In addition to these four observables, we consider the specific combination $h(z)$ that is probed by the AP geometrical test. AP pointed out that while tests using $d_{A}(z)$ or $d_{L}(z)$ can be affected by evolution in the sizes of "standard rulers" or the luminosities of "standard candles," one can measure the ratio of redshift separation distance to angular separation distance assuming only that the structures under investigation are isotropic. Recent implementations of this idea 
consider, instead of the idealized spherical clusters discussed by AP, the statistical pattern of clustering traced by quasars (Phillips 1994; Ballinger, Peacock, \& Heavens 1996; Matsubara \& Suto 1996; Popowski et al. 1998; Outram et al. 2001; Calvão et al. 2002), galaxies (Ryden 1995; Nakamura, Matsubara, \& Suto 1998; Nair 1999; Matsubara \& Szalay 2001) or the Ly $\alpha$ forest (Hui et al. 1999; McDonald \& MiraldaEscudé 1999; McDonald 2001). Adopting the notation of Phillips (1994) and Popowski et al. (1998), we define

$$
h=\frac{\Delta z}{z \Delta \theta}
$$

as the ratio of redshift separation to a "redshift arc length" for equal tangential and line-of-sight separations in physical coordinates, assuming $\Delta z \ll z$. For a fixed physical separation, $\Delta z$ is proportional to $H(z)$ and $(\Delta \theta)^{-1}$ is proportional to $d_{A}(z)$, so $h(z)$ is proportional to their product,

$$
h(z)=\frac{1+z}{c z} H(z) d_{A}(z) .
$$

We will refer to $h(z)$ as the AP parameter.

What are the prospects for measuring these observables in the next 5-10 yr? Our remarks here will be qualitative and somewhat speculative, but it is useful to approach the predictions of $\S 4$ with some sense of what may be achieved by different methods.

The prospects for distance measurements are the clearest and most well studied. The rms scatter of the relation between peak luminosity and light-curve shape for SNe Ia is only $\sim 10 \%$ (Phillips 1994; Riess, Press, \& Kirshner 1996), so each well-observed supernova allows a distance estimate with a $1 \sigma$ statistical uncertainty $\sim 5 \%$. Current samples (Hamuy et al. 1996; Riess et al. 1998; Perlmutter et al. 1999) are concentrated at $z \sim 0$ and $z \sim 0.5-0.8$, but the approach can be extended to $z \sim 1.7$ if the $S N A P$ satellite is built. With samples of hundreds or even thousands of supernovae, the statistical errors will become very small indeed, and the measurement accuracy is likely to be limited by systematic uncertainties such as dust extinction, possible evolution of the progenitor population, and stability of photometric calibration over a wide dynamic range. A precision $\sim 1 \%$ to $z \sim 1.7$ seems plausibly achievable and perhaps even unduly pessimistic.

There are numerous other ways to measure the distanceredshift relation. Measurements of the Sunyaev-Zeldovich decrement and X-ray properties of clusters can be combined to yield the angular diameter distance (Birkinshaw 1999; Molnar, Birkinshaw, \& Mushotzky 2002). This method can provide an entirely independent check on SN Ia results, and its limiting precision depends on the size of well-observed cluster samples and the accuracy with which internal cluster properties (particularly substructure) can be understood. The angular diameter distance can also be measured by identifying a characteristic scale (such as the curvature scale of the CDM power spectrum) in the angular clustering of distant clusters (Cooray et al. 2001) or even by using the amplitude of this angular clustering in comparison to theoretical predictions. Roukema \& Mamon (2000) have already applied a similar approach to a putative feature at $\sim 130 h^{-1}$ $\mathrm{Mpc}$ in the quasar power spectrum. Galaxy counts in a deep redshift survey depend on the volume element $d V \propto d_{A}^{2}(z) H^{-1}(z)$, providing yet another way to constrain distances. The chief uncertainty in this approach is evolu- tion of the galaxy population, but Newman \& Davis (2000, 2002) argue that this can be controlled in the DEEP redshift survey by measurement of galaxy circular velocities, allowing useful constraints on $w$.

At redshifts $z \gtrsim 2$ all of these methods become difficult, but the $\operatorname{Ly} \alpha$ forest offers an alternative probe out to $z \sim 4$. The predicted correlation of flux along lines of sight to quasar pairs depends on $d_{A}(z)$, and measurements of this correlation will improve as more close pairs are discovered and studied. Such estimates of $d_{A}(z)$ would be somewhat modeldependent, but the statistics of flux along individual lines of sight can provide detailed checks of the assumed model. This method has not been investigated in any detail (although McDonald 2001 presents relevant results), so it is hard to know what precision can be achieved, perhaps a few percent. Strong gravitational lensing statistics also test the equation of state through their dependence on distance at various redshifts (Cooray \& Huterer 1999), and constraints can also be obtained by measuring the source redshifts in well-understood lens systems (Yamamoto et al. 2001). Finally, the first acoustic peak in the CMB power spectrum gives a high-precision measurement of the angular diameter distance to the surface of last scattering, at $z=z_{r} \approx 1100$. The uncertainty in this determination is associated with the uncertainty in the parameters that determine the sound horizon at $z_{r}$, which are themselves constrained by the CMB power spectrum and other cosmological observations. It is again hard to know just what precision will be obtained on $d_{A}\left(z_{r}\right)$ itself, but a percent or better seems plausible.

Out to redshift $z \sim 1$, the main observational probe of the growth factor $D_{1}(z)$, at least at present, is the mass function of galaxy clusters. Because clusters are rare objects that form from 2-3 $\sigma$ excursions of the initial Gaussian fluctuation spectrum, their predicted abundance is sensitive to the normalization of that spectrum and thus to the product $\sigma_{8} D_{1}(z)$, where $\sigma_{8}$ is the rms fluctuation of matter in spheres of radius $8 h^{-1}$ Mpc at $z=0$ (see, e.g., Bahcall et al. 1997). However, because the cluster mass function is steep, abundances are also sensitive to the accuracy and precision of mass determinations (Frenk et al. 1990), and the limitation on measurements of $D_{1}$ is likely to be systematic rather than statistical. The combination of X-ray, Sunyaev-Zeldovich, and weak-lensing approaches should reduce these systematic uncertainties below current levels. To guess what level of precision is achievable for $D_{1}$, we note that current " $2 \sigma$ " uncertainties in the fluctuation amplitude $\sigma_{8}$ (for specified $\Omega_{m, 0}$ ) are $\sim 10 \%$ (see, e.g., Eke, Cole, \& Frenk 1996), although independent estimates can differ by more than this amount even when the input data are similar (see, e.g., Seljak 2001). Balancing the difficulties of working at higher redshift against the anticipated large improvements in cluster data, it seems reasonable to hope for $\sim 5 \%$ precision in $D_{1}$ out to $z \sim 1$, possibly better. Recent discussions of the potential of galaxy cluster surveys for constraining $w$ include Haiman et al. (2001), Newman et al. (2002), and Weller et al. (2001).

Cosmic shear is another potential probe of $D_{1}(z)$, measuring the amplitude of surface density fluctuations (see Huterer 2002 for a discussion in the context of $w$ constraints). Recent measurements already yield a constraint on $\sigma_{8}$ (for fixed $\Omega_{m, 0}$ ) that is competitive with determinations from the cluster mass function, with remarkably good agreement of independent estimates (see Maoli et al. 2001 and references therein). Measurements of shear for samples 
of foreground and background galaxies with different photometric redshifts should allow $\sigma_{8}$ and $D_{1}(z)$ to be disentangled. Weak lensing will, at the least, provide an independent check on estimates of $D_{1}(z)$ from cluster masses, and the ambitious surveys now underway may eventually yield significantly better precision. At $z>2$, the most promising route to $D_{1}(z)$ is the flux power spectrum of the Ly $\alpha$ forest, which is related to the underlying matter power spectrum in a fairly straightforward way (Croft et al. 1998, 1999, 2001; McDonald et al. 2000; Gnedin \& Hamilton 2001; Zaldarriaga, Hui, \& Tegmark 2001). Current uncertainties in the rms fluctuation amplitude are $\sim 15 \%$, with roughly equal statistical and systematic uncertainties. The former will decrease with larger samples such as those from the Sloan Digital Sky Survey (York et al. 2000), while the latter will decrease with improved determinations of the mean Ly $\alpha$ flux decrement, improved numerical simulations to calibrate the relation between the flux and matter power spectra, and the use of other statistics to test the assumptions that enter these simulations. At this point, it is not clear where systematic uncertainties will limit the precision of mass fluctuation measurements from the Ly $\alpha$ forest, but $5 \%-10 \%$ seems a reasonable guess. The other redshift at which we can expect to determine $D_{1}(z)$ is the redshift of recombination from comparing the amplitude of the $\mathrm{CMB}$ power spectrum to that of today's matter power spectrum (Doran et al. 2001). Here measurement precision will be high, and the limiting factor is the degeneracy of the fluctuation amplitude with other parameters that affect the level of CMB anisotropy.

We note in passing that the cluster abundance, cosmic shear amplitude, and $\operatorname{Ly} \alpha$ flux power spectrum are not "pure" measurements of $D_{1}(z)$ since the distance-redshift relation affects the first two through volume factors and lensing geometry, respectively, and the Hubble parameter affects the third because the power spectrum is measured in kilometers per second at the observed redshift. Similarly, the angular diameter distance is needed to identify angular scales in the CMB with length scales at $z=0$. However, given the direct dependence of these quantities on the mass fluctuation amplitude, it makes sense to describe them primarily as probes of the growth factor. Going from an amplitude of fluctuations at redshift $z$ to a value of $D_{1}(z)$ also requires accurate knowledge of the fluctuation amplitude today (i.e., of $\sigma_{8}$ ), which we are implicitly assuming will emerge from the tightening web of $\mathrm{CMB}$, large-scale structure, cluster, and weak-lensing constraints. The obtainable precision on $D_{1}$ may be higher than the precision in $\sigma_{8}$ itself in the case of a differential evolutionary measurement, such as the cluster mass function.

Lower limits to the age of the universe can be obtained by modeling the stellar populations of the oldest galaxies observed at a given redshift. This approach has been used to argue against $\Omega_{m}=1$ models, for which the age scales as $t(z)=t_{0}(1+z)^{-3 / 2}$; even relative to open models, the addition of a cosmological constant makes it substantially easier to understand the red colors and high stellar mass-to-light ratios of high-redshift elliptical galaxies (see, e.g., Peacock et al. 1998; van Dokkum et al. 1998). Lima \& Alcaniz (2000) have investigated the usefulness of galaxy ages as a constraint on $w$. Given the uncertainties associated with population synthesis modeling and dust extinction, precision of $10 \%$ or better in $t(z)$ at high $z$ would seem highly optimistic. However, age constraints can provide an upper limit on $w$ that allows a consistency check with other estimates. Exploiting this limit requires accurate knowledge of $H_{0}$, which sets the overall normalization of timescales.

The most promising targets for the AP test are quasars (Phillips 1994; Ballinger et al. 1996; Matsubara \& Suto 1996; Popowski et al. 1998; Outram et al. 2001), the Ly $\alpha$ forest toward quasar pairs (Hui et al. 1999; McDonald \& Miralda-Escudé 1999; McDonald 2001), and galaxies in the Sloan or 2dF redshift surveys (Ryden 1995; Nakamura et al. 1998; Matsubara \& Szalay 2001). The Ly $\alpha$ forest approach is elegant, but McDonald (2001) shows that $h(z)$ at $z \sim 2-4$ is insensitive to $w$, and our results below reinforce this conclusion. Instead, $h(z)$ at these redshifts provides a good diagnostic of $\Omega_{m, 0}$ (and thus $\Omega_{\phi, 0} \approx 1-\Omega_{m, 0}$ ), with little dependence on $w$ if it is less than -0.5 (McDonald 2001). A precise value of $\Omega_{m, 0}$ is needed to get useful constraints on $w$ with other tests, as we discuss and illustrate below. Most studies of the AP test with quasars or galaxies also focus on $\Omega_{m, 0}$ and $\Omega_{\Lambda, 0}$ rather than $w$. However, Calvão et al. (2002) have examined constraints on $w$ that could be obtained with the $2 \mathrm{dF}$ quasar redshift survey with encouraging conclusions. They do not present their results in the form of precision on $h(z)$, but their projected sensitivity to $w$ must imply fairly good precision at $z \sim 0.5-1$.

The Hubble parameter $H(z)$ is the observable most directly tied to the Friedmann equation (1). One way to measure it is by combining the volume-redshift or AP test with estimates of $d_{A}(z)$. The Ly $\alpha$ forest offers a more direct route because the width and separation of features is determined largely by Hubble flow (Weinberg et al. 1997). Statistics like the threshold-crossing frequency are sensitive to the difference between open and flat CDM models because of the difference in $H(z)$ (Weinberg et al. 1999a), and measurements of the power spectrum shape can yield characteristic scales in kilometers per second at the observed redshift for comparison with scales measured in units of $h^{-1} \mathrm{Mpc}$ at $z=0$ (Croft et al. 2001). This method of measuring $H(z)$ has not been investigated in any detail, so we do not know what precision is attainable; it is likely to be set by the trade-off between $H(z)$ and other parameters that describe the temperature-density relation of the diffuse intergalactic medium. It is likely to work better at $z \sim 2-4$ than at lower redshifts, where the observations must be done from space, and shock-heated gas contributes more to the Ly $\alpha$ forest (Davé et al. 1999), although even here the separation between features might prove a useful diagnostic of the expansion rate.

At $z \lesssim 1$, the skewness of the cosmic shear distribution offers an alternative probe of $H(z)$. Hui (1999) discusses the constraints on $w$ that can be obtained by this method, which arise from the sensitivity of the predicted skewness to the value of $\Omega_{m}(z)$. Since the matter density is necessarily $\rho_{m}(z)=\Omega_{m, 0} \rho_{c, 0}(1+z)^{3}$, the cosmology dependence of $\Omega_{m}(z)$ comes from the critical density $\rho_{c}(z)=3 H^{2}(z) / 8 \pi G$, so in the context of our discussion it makes sense to view weak-lensing skewness as a measurement of $H(z)$. Jimenez $\&$ Loeb (2001) have proposed yet another route to measuring $H(z)$, using the relative ages of galaxy populations at two different redshifts (which can be determined more accurately than the absolute ages since some of the uncertainties in the population synthesis models cancel out). The ratio of redshift difference to age difference yields $d z / d t=-(1+z) H(z)$, where the equality uses the definitions $(1+z)=a_{0} / a$ and $H=\dot{a} / a$. Note that while the $\operatorname{Ly} \alpha$ 
forest and weak-lensing methods effectively measure the ratio $H(z) / H_{0}$, the age difference method gives $H(z)$ in physical units. Jimenez \& Loeb (2001) argue that percent-level precision in $H(z)$ is achievable, in which case the uncertainty in the ratio (which is the quantity sensitive to the equation of state) is likely to be dominated by the uncertainty in $H_{0}$ itself.

There is significant degeneracy between the value of $\Omega_{m, 0}$ and the value of $w$ since either lower $\Omega_{m, 0}$ or lower $w$ leads to greater acceleration. We assume that improving $\mathrm{CMB}$ and large-scale structure measurements will allow a precise determination of $\Omega_{m, 0}$ in the next few years, independent of measurements of $d_{A}, D_{1}, t, H$, and $h$, so that the power of these constraints can be brought to bear entirely on the equation of state. We will consider the impact of a 0.05 uncertainty in the value of $\Omega_{m, 0}$, and it is not obvious whether this assumption is optimistic or pessimistic. Apart from the determination of $\Omega_{m, 0}$, the only role that we ascribe to the CMB is the measurement of $d_{A}$ and $D_{1}$ at $z \approx 1100$. It may be that CMB data can also yield constraints on the expansion history and $D_{1}$ at lower redshifts, via the integrated Sachs-Wolfe (1967) effect or lensing of anisotropies (see, e.g., Seljak 1996), but we do not know just what these constraints will be. If the quintessence field is inhomogeneous, it will contribute to large-angle CMB anisotropy (Caldwell et al. 1998), allowing a probe of the dark energy independent of the ones considered here, which are all based on the expansion history.

By focusing on this specific set of observables, we do not wish to imply that this is necessarily the ground on which theory and observation will be compared. Presumably, the constraints on the equation of state from, say, weak lensing will be derived in terms of the weak-lensing observables themselves without first extracting constraints on $D_{1}, d_{A}$, and $H$ at various redshifts. However, in trying to understand the potential power of combining different observational approaches, it is helpful to think in terms of the fundamental quantities that they can measure. In particular, two models that predict indistinguishable results for $d_{A}(z)$, $D_{1}(z), t(z)$, and $H(z)$ cannot be discriminated by any combination of observations that depends only on these quantities. Our focus on fundamental observables is also a helpful way of estimating the level of precision needed for some observational strategy to make a useful contribution to constraining the equation of state and its history.

\section{DEPENDENCE OF THE OBSERVABLES ON THE EQUATION OF STATE}

Figure 3 presents our basic results for the quintessence models discussed in $\S 2$. Each panel shows the evolution of one of the five observables, $d_{A}, D_{1}, t, H$, or $h$, out to redshift $z=10^{3} \approx z_{r}$. Open triangles and squares represent constant- $w$ models with $n=1$ and $n=2\left(w=-\frac{2}{3}\right.$ and $\left.-\frac{1}{3}\right)$, respectively. Filled triangles and squares represent $V=\phi^{-1}$ and $V=\phi^{-6}$ models, respectively, and stars represent the Albrecht-Skordis model with the parameters stated in $\S 2$. In all cases, the points are computed assuming a flat universe, $\quad \Omega_{m, 0}=0.4, \quad \Omega_{r, 0}=9.8 \times 10^{-5}, \quad$ and $\Omega_{\phi, 0}=1-\Omega_{m, 0}-\Omega_{r, 0}$. Furthermore, we normalize the value of each observable to the value predicted by a pure- $\Lambda$ model $(n=0, w=-1)$ at the corresponding redshift. Thus, for example, the open triangle at $z=0.5$, $d_{A}(z) / d_{A}(z)_{\Lambda}=0.96$ implies that a precision of $4 \%$ (at the desired confidence level) is sufficient to distinguish an $n=1$ model from a $\Lambda$ model using the angular diameter distance at $z=0.5$ if $\Omega_{m, 0}$ is known perfectly. The ends of the error bars on each point show results for models with the same equation of state but $\Omega_{m, 0}=0.35$ and 0.45 to illustrate the impact of uncertainty in $\Omega_{m, 0}$. (Ratios are still computed relative to an $\Omega_{m, 0}=0.4 \Lambda$ model.) If the error bar on an observable overlaps a ratio of 1.0 , then even a perfect measurement of that observable at that redshift will not distinguish the model from a $\Lambda$ model unless $\Omega_{m, 0}$ is known to better than 0.05 .

For a given precision, the sensitivity of different observables peaks at different redshifts. The Hubble parameter sensitivity peaks at $z \sim 1-2$, when the ratio of $\Omega_{\phi}(z)$ values in different models is large and the quintessence energy density is still large enough to be dynamically important (see Fig. 2; roughly speaking, it is the absolute difference in $\Omega_{\phi}$ in this figure that matters for differences in $H$ ). The sensitivity of $d_{A}(z)$ remains fairly flat since even at high $z$ the distance "remembers" the behavior of $H(z)$ at low redshifts (see eq. [11]). The age of the universe, by contrast, depends only on the Hubble parameter at redshifts higher than $z$ (eq. [10]), so the sensitivity of $t(z)$ continues to increase almost all the way down to $z=0$. Note that the sensitivity of an observable to the value of $n$, displayed in Figure 3, may be quite different from the sensitivity of that observable's $z$-derivative, which often peaks at lower redshift.

The behavior of $h(z)$ is governed by the competing effects of $H(z)$ and $d_{A}(z)$-from equations (16) and (11), one can see that $h(z)$ is proportional to the product of $H(z)$ and the average value of $H^{-1}(z)$ at lower redshifts. At $z \approx 3, h(z)$ is very insensitive to $w$, as pointed out by McDonald (2001), who emphasizes that this independence of $w$ makes the AP test at this redshift an especially good diagnostic of $\Omega_{m, 0}$. The sensitivity to $w$ peaks at $z \sim 0.5$, making quasar clustering better than the Ly $\alpha$ forest as a probe of the equation of state per se. The Sloan survey's luminous red galaxy sample (Eisenstein et al. 2001) might also be useful for this application (Matsubara \& Szalay 2001). The sensitivity of $h(z)$ to $w$ grows again at $z \gtrsim 5$, but the prospects for applying the AP test at these redshifts seem very slim.

The sensitivity of $D_{1}(z)$ increases with increasing $z$ since the growth factor depends only on clustering between redshift $z$ and redshift zero, and models with lower $n$ have larger $\Omega_{m}(z)=1-\Omega_{\phi}(z)-\Omega_{r}(z)$ at all $z>0$. For $n=1$, the sensitivity levels out at $z \gtrsim 3$ as quintessence becomes dynamically unimportant, but for $n=2$, the value of $\Omega_{\phi}(z)$ is nonnegligible even at fairly high redshift (Fig. 2).

Figure 3 shows that $\sim 10 \%$ measurements of any of these observables near their redshift of peak sensitivity can discriminate an $n=2$ model from a $\Lambda$ model or from an $n=1$ model. Since the expansion of an $n=2$ model with $\Omega_{m, 0}>0$ is always decelerating, it is not surprising that this model is fairly easy to distinguish from a $\Lambda$ model with significant acceleration at low redshift. SN Ia measurements already rule out this value of $n$ (Garnavich et al. 1998), and Figure 3 implies that other observations are within reach of confirming this result independently. Distinguishing an $n=1$ model from a $\Lambda$ model is much harder, typically requiring measurement precision of a few percent or better and independent precision on $\Omega_{m, 0}$ that is not much worse than the 0.05 represented by our error bars. Nonetheless, this level of discrimination is clearly within reach of the improving SN Ia 

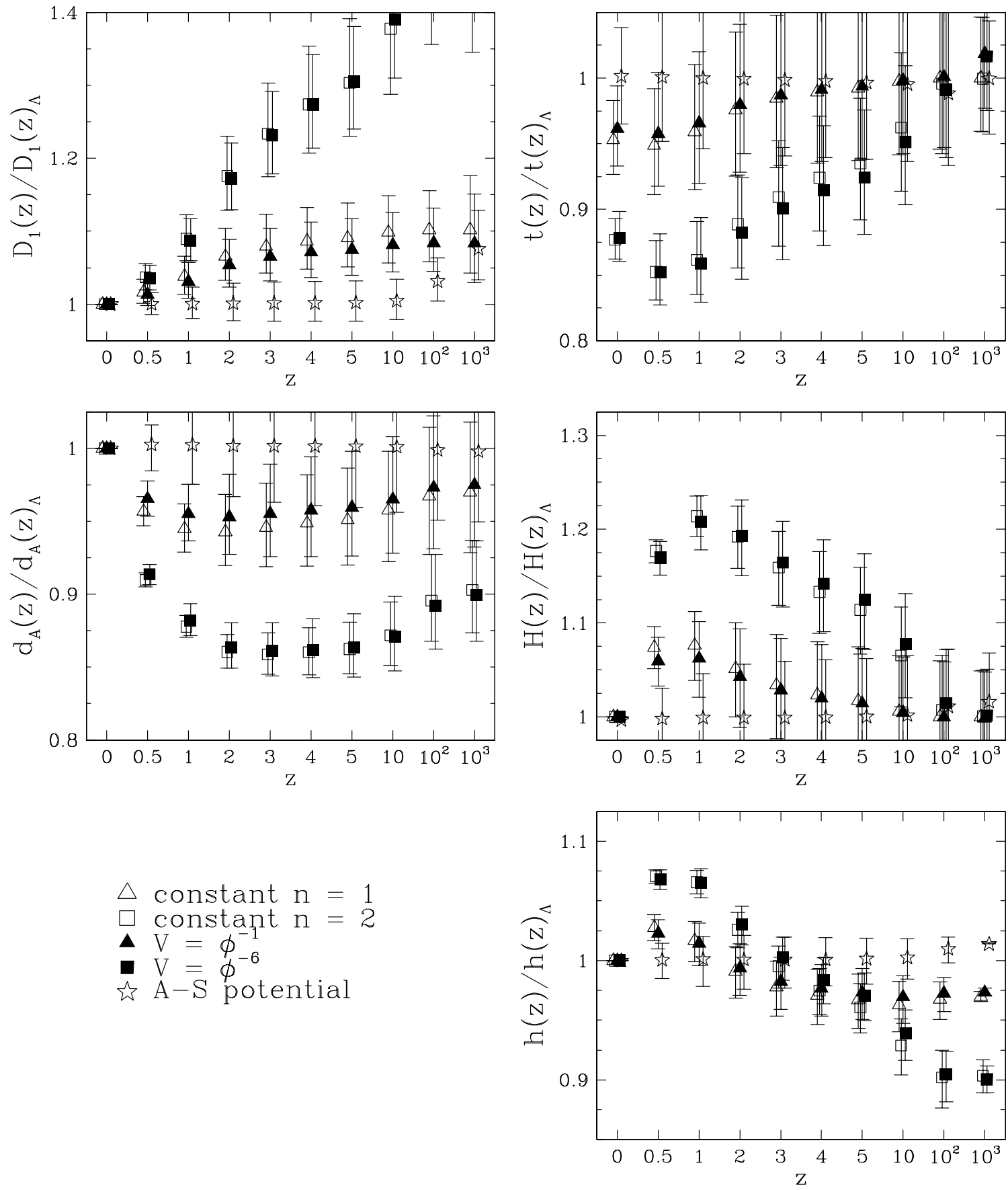

FIG. 3. $-D_{1}(z), t(z), d_{A}(z), H(z)$, and $h(z)$ vs. $z$ for the constant $n=1,2$ models, the fixed potential models $V=\phi^{-1}, \phi^{-6}$, and the Albrecht-Skordis potential. The central value represents $\Omega_{m, 0}=0.4$, and the error bars are at $\Omega_{m, 0}=0.35,0.45$. Quantities are normalized to the $\Lambda$ model with $\Omega_{m, 0}=0.4$. A small horizontal offset has been added to the points to allow them to be distinguished. The $\Omega_{m, 0}=0.45$ end of the error bar is usually the end farther from a ratio of unity (or for the Albrecht-Skordis model, the end closer to the $n=1$ points) except for $D_{1}(z)$ and the high-redshift $(h<1)$ regime of $h(z)$, where the $\Omega_{m, 0}=0.45$ end is closer to unity.

measurements, and the discussion in $\S 3$ suggests that several other methods have a realistic hope of reaching the necessary precision on a timescale of several years. The sensitivity of our observables to $w$ would be slightly greater if we adopted $\Omega_{m, 0}=0.3$ as our central value. Furthermore, the precision that might be obtained from measurement of these observables at multiple redshifts can be higher if the errors in the separate measurements are uncorrelated; however, if the source of uncertainty is systematic, it may produce correlated errors at different redshifts.
Unfortunately, distinguishing any of the time-varying $w$ models that we have considered from the closest constant- $w$ model looks all but impossible. The $V=\phi^{-1}$ model tracks the $n=1$ model almost perfectly, and the $V=\phi^{-6}$ model tracks the $n=2$ model with similar faithfulness. The close match of these models is unsurprising given the plots of $n(z)$ and $\Omega_{\phi}(z)$ in Figures 1 and 2. The predicted differences between the power-law potential models and the constant- $n$ models and the redshift dependence of these differences have the sign one would expect from Figure 1; the problem is sim- 
ply that the time dependence of the equation of state predicted by these models is extremely weak. Similarly, the Albrecht-Skordis model is virtually indistinguishable from a pure- $\Lambda$ model because it has $n \approx 0$ at all redshifts where quintessence is dynamically important, even though it has a very different $n$ at $z>3$. The one potential distinguishing feature of the Albrecht-Skordis model is the value of $D_{1}$ at recombination, which is about $7.5 \%$ larger than that of a $\Lambda$ model. This level of precision is plausibly within reach of future observations. The distinguishability of the AlbrechtSkordis model would increase if the equation-of-state transition were shifted toward lower redshift and vice versa.

Since we have so little empirical information about the nature of dark energy, there is no reason to think that models presently in the literature exhaust the possibilities for the time dependence of the equation of state. We have therefore constructed a set of "toy" models that exhibit a wider range of behavior so that we can better understand the ability of observations to detect time variation if it is present. For these models, we assume that the redshift dependence of $\rho_{\phi}$ is a broken power law of the form

$$
\rho_{\phi}(z)=\frac{\left(1+z_{c}\right)^{n-m}(1+z)^{n}}{\left(1+z_{c}+z\right)^{n-m}} \rho_{\phi}(z=0)
$$

where $z_{c}$ is the critical redshift near which the scaling behavior of $\rho_{\phi}$ changes from $(1+z)^{m}$ for $z \gg z_{c}$ to $(1+z)^{n}$ for $z \ll z_{c}$. The model therefore switches from an early-time constant- $m$ case to a late-time constant $n$ case. This class of models allows us to examine the effects of a more extreme change in the power-law scaling of $\rho_{\phi}$ than is exhibited by the tracker models examined earlier. (For the cases $m=n$ or $z_{c}=0$, this model reduces exactly to the constant $n$ models discussed earlier, while the $m=3, n=0$ case resembles the Albrecht-Skordis model.) Note that a similar but somewhat different toy model was examined by Huterer \& Turner (2001), who looked at models in which $w$ was taken to be constant in discrete redshift bins.

Figure 4 shows $\Omega_{\phi}(z)$ for a variety of broken power-law models. The late-time behavior $(n)$ has been fixed at $n=1$, and early-time behavior $(m)$ has been taken to be $m=0$ or $m=2$ for two different values of the critical redshift $\left(z_{c}=1\right.$ and $z_{c}=3$ ). For comparison, we have also included three constant- $n$ models $(m=n)$, namely, $m=n=0,1$, and 2 . As expected, $\Omega_{\phi}(z)$ in our broken power-law models deviates from its behavior in the constant $n$ models to a much greater extent than is the case for the power-law potentials in Figure 2.

In Figures 5-7 we examine our five observables for the broken power-law cases $m, n=0,1,2$ and $z_{c}=1$, 3. Each panel of graphs shows models with a different late-time behavior (different values of $n$ ), and the observables in each panel are normalized to the corresponding constant- $n$ case: $n=0$ in Figure 5, $n=1$ in Figure 6, and $n=2$ in Figure 7. The deviation from a ratio of unity in each case shows the observational effect of the break in scaling behavior. This deviation is quite significant in many cases, often more than $10 \%$ for $H, d_{A}$, or $t$ and up to almost $30 \%$ for $D_{1}$. The broken power-law model is therefore clearly distinguishable from the constant $-n$ model that has the same value of $n$ at $z=0$.

However, if we choose a constant- $n$ model that is matched to the "average" behavior of the broken power-law model instead of the $z=0$ value of $n$, then this distinguishability vanishes. The curves in Figures 5-7 show, for each broken

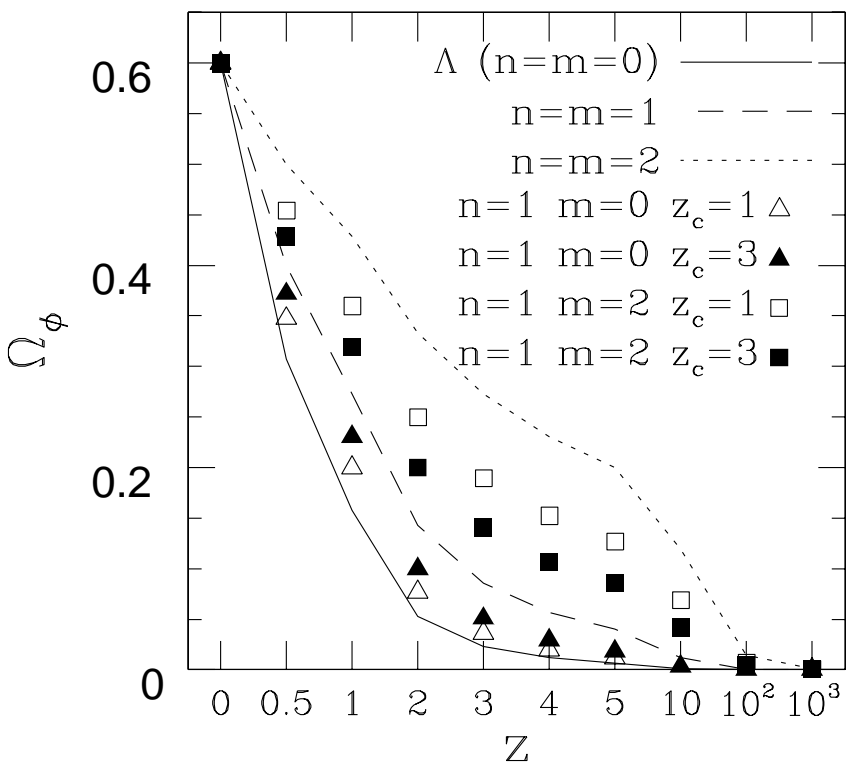

FIG. 4. $-\Omega_{\phi}$ as a function of redshift $z$ for the indicated broken powerlaw models, defined by eq. (17). The curves show three constant- $n$ models for reference.

power-law case, the predictions of a constant- $n$ model selected to produce the same value of the Hubble parameter $H(z)$ at $z=1$. This matched constant- $n$ model predicts nearly the same values for all observables at every redshift as the corresponding broken power-law model. By design, our toy model has a large change in $n$ (and thus $w$ ) at low redshift, but this time variation cannot be detected unless the observables can be measured to extremely high precision. Making the transition redshift $z_{c}$ higher or lower does not make the time dependence easier to detect; it just changes the effective average value of $n$, so the constant- $n$ model that matches the broken power-law model is different from before. For example, we have constructed models with $z_{c}=0.5$, and our results are quite similar, except that the corresponding constant $-n$ model has a value of $n$ that is closer to the low-redshift exponent in the broken power-law model. A sharper transition would be easier to detect, but our models already change the energy scaling index by order unity over a redshift interval $\Delta z \sim z_{c}$ [and thus a time interval $\Delta t \sim H^{-1}(z)$ ], and a much faster transition seems physically unlikely, although such models can be constructed (see, e.g., Weller \& Albrecht 2001).

While our results suggest that generic variations of the equation of state with redshift are essentially undetectable, there is one exception to this rule that is worthy of note. Our models with high-redshift index $m=2$ predict values of $D_{1}(z)$ at $z=10^{3}$ that differ by a few percent from those of the constant- $n$ model that matches the lowredshift observables. This level of precision might plausibly be achieved with comparisons of CMB anisotropy to local clustering, although substantial improvements in observational data would be required. [The values of $t(z)$, $H(z)$, and $h(z)$ also show percent-level deviations from the matched constant $-n$ model at $z \gtrsim 3$, but we see no plausible routes to attaining the necessary precision for these quantities.] The behavior of the $m=2$ models is reminiscent of the Albrecht-Skordis model, and the cause is similar: the energy density of the quintessence compo- 

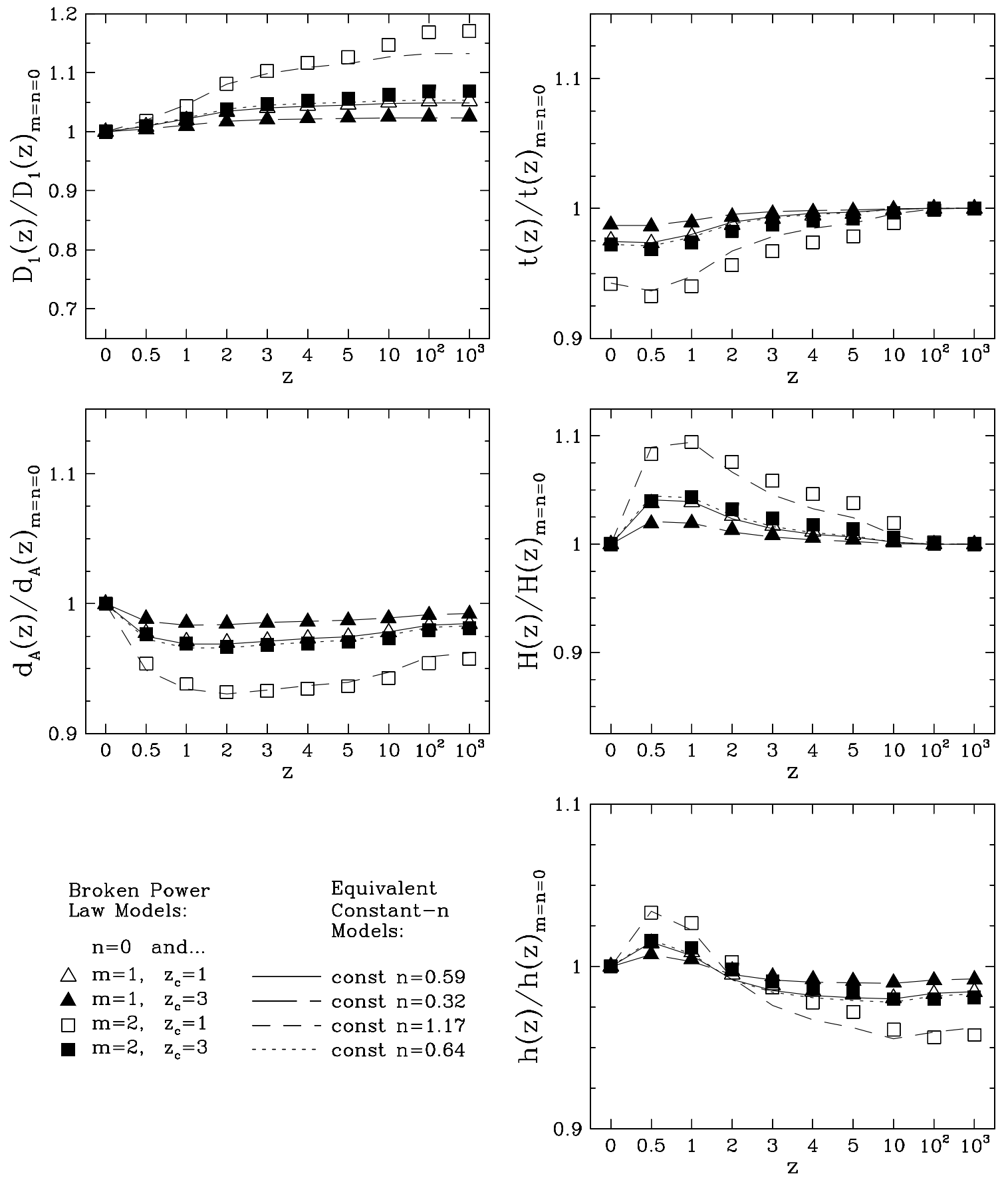

FIG. 5.- $D_{1}(z), t(z), d_{A}(z), H(z)$, and $h(z)$ vs. $z$ for four broken power-law models, all having the same late-time behavior, $n=0$. The points are normalized to the value of the given observable in the $n=0$ model at the same redshift. For each set of points, a constant- $n$ model that has the same value of $H(z)$ at $z=1$ is shown.

nent remains a nonnegligible fraction of the critical density out to fairly high redshift, so the gravitational growth of matter clustering is correspondingly slower.

\section{CONCLUSIONS}

Our results both confirm and extend earlier work on this subject. For any of the five observable quantities considered here - the angular diameter distance $d_{A}(z)$, the Hubble parameter $H(z)$, the age of the universe $t(z)$, the linear growth factor $D_{1}(z)$, or the Alcock-Paczynski parameter
$H(z) d_{A}(z)$-measurement with $\sim 10 \%$ precision near the observable's redshift of peak sensitivity would be sufficient to distinguish an $n=2\left(w=-\frac{1}{3}\right)$ model from a pure cosmological constant, even if $\Omega_{m, 0}$ were known only to an accuracy of \pm 0.05 . Although this value of $w$ is already ruled out by the SN Ia measurements, our results suggest that other observations may soon be able to independently confirm the result. Distinguishing an $n=1\left(w=-\frac{2}{3}\right)$ model from a pure cosmological constant is much harder, demanding measurement precision of a few percent near the redshift of peak sensitivity, along with a determination of $\Omega_{m, 0}$ to within 

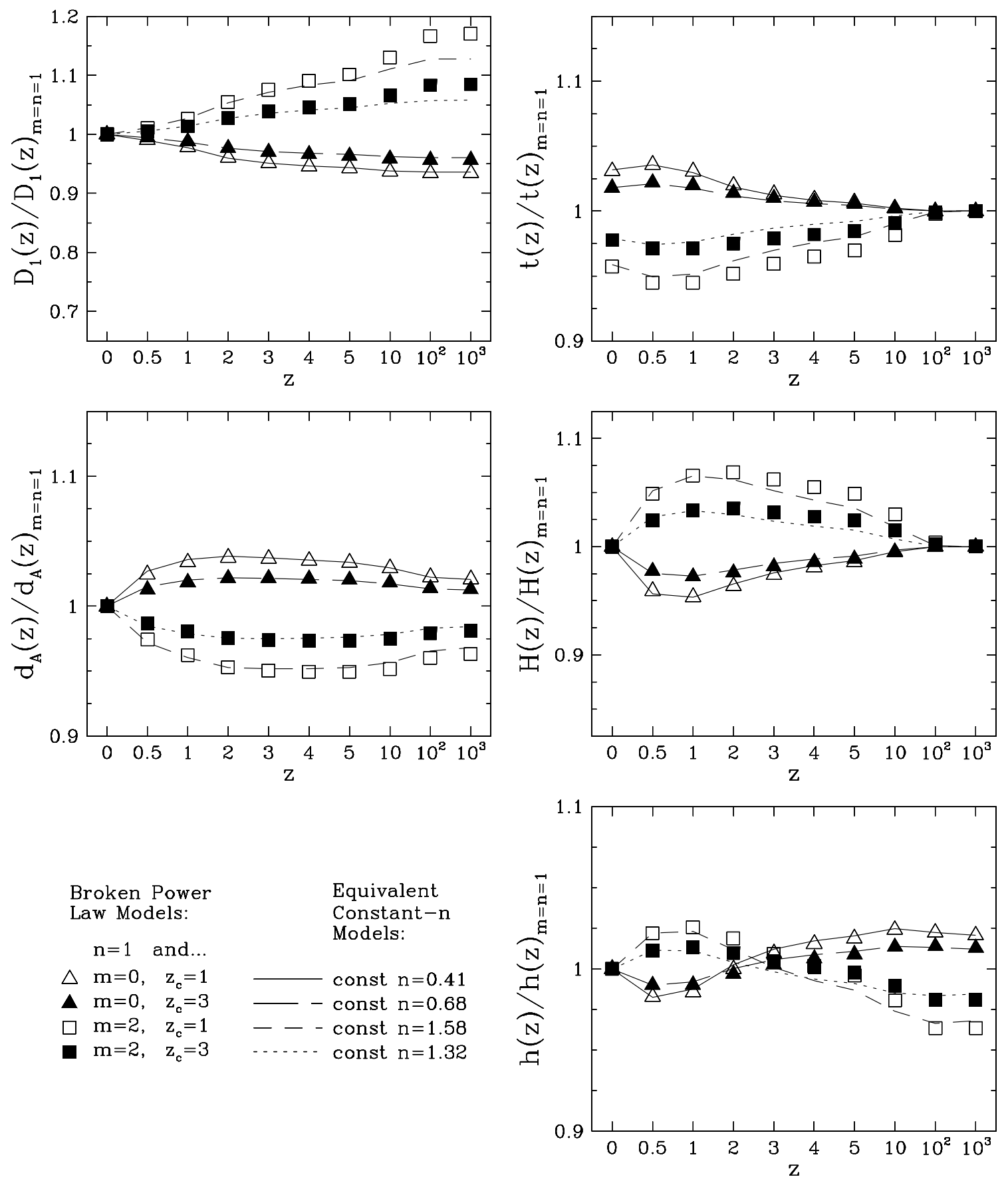

Fig. 6. - Same as Fig. 5, but for the late-time behavior $n=1$. The points are normalized to the $n=1$ model.

\pm 0.05 . Although this level of precision is currently unavailable, it seems clearly within reach of improving SN Ia data, and it is likely to be achieved by one or more of the other observational methods discussed in $\S 3$. Thus, while SN Ia surveys may provide the first precise determination of $w$, a collection of other observations seems likely to provide confirmation (or refutation!) of the measurement within a few years.

The sensitivity of the observables to the value of $n$ depends on redshift in different ways, reflecting the links between these quantities and the expansion history. The age $t(z)$ depends only on expansion at redshifts greater than $z$, so its sensitivity to $n$ decreases monotonically with increas- ing $z$. The linear growth factor, on the other hand, depends on clustering from redshift $z$ to redshift zero, so the sensitivity of $D_{1}(z)$ increases monotonically with $z$. The Hubble parameter $H(z)$ is most sensitive at $z \sim 1-2$, when $\Omega_{\phi}$ is substantially different from its present-day value but not so small that quintessence is dynamically unimportant. The sensitivity of the angular diameter distance is fairly flat over a wide range of redshifts. The sensitivity of the AP parameter is governed by competing effects of $H(z)$ and $d_{A}(z)$, which cancel each other at $z \sim 3$.

Because of their different connections to the expansion history, we hoped at the outset of this investigation that these observables would provide complementary informa- 

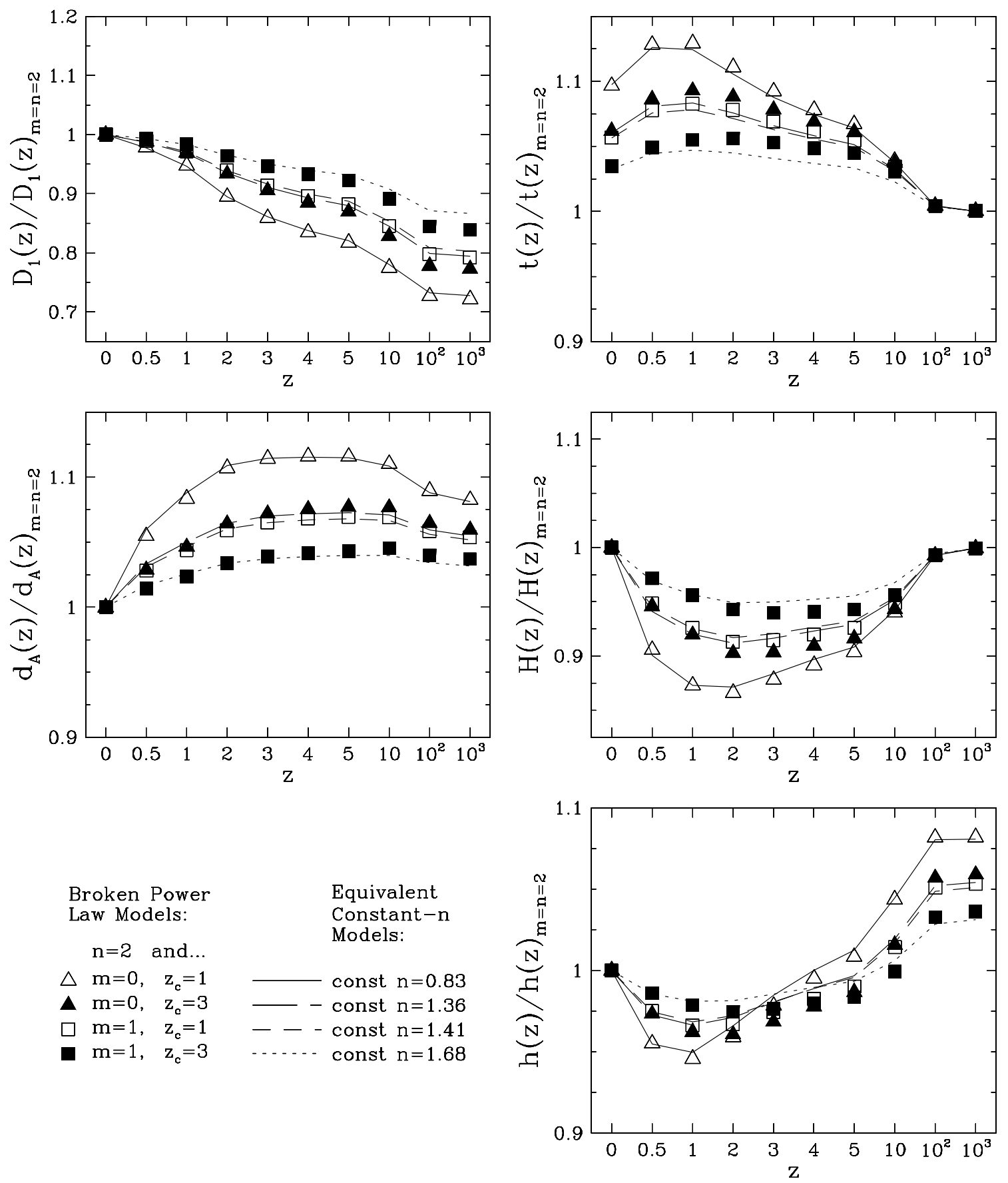

FIG. 7.- Same as Fig. 5, but for the late-time behavior $n=2$. The points are normalized to the $n=2$ model.

tion about the history of the equation of state, allowing a combination of measurements to detect a time variation of $w$ that could not be found by any one method on its own. Unfortunately, we find that the level of complementarity is too weak to be useful in practice: models that make indistinguishable predictions for one observable generally make indistinguishable predictions for all of them. Of course, it is valuable to confirm an important result like a measurement of $w$ by independent methods to check for systematic errors or a breakdown of the assumptions implicit in each approach. Furthermore, different observables can provide complementary information about $\Omega_{m, 0}$, precise knowledge of which is essential if one hopes to constrain $w$. However, once $\Omega_{m, 0}$ is known, the constraints on the equation of state and its history will be dominated by the single highest precision measurement; adding lower precision measurements of other observables will give little additional purchase.

We find, furthermore, that none of the observables hold much promise for distinguishing a quintessence model with a time-dependent equation of state from an appropriately chosen constant $n$ model, even if one is highly optimistic about the achievable precision and assumes perfect, independent knowledge of $\Omega_{m, 0}$. Tracker models with $V(\phi) \propto \phi^{-1}$ and $V(\phi) \propto \phi^{-6}$ are effectively identical to models with constant $n=1$ and $n=2$, respectively. Models with an Albrecht-Skordis potential cannot be distinguished 
from a pure- $\Lambda$ model, except, perhaps, by a measurement of the growth factor at recombination from CMB anisotropy (a point we will return to shortly). The fundamental difficulty is that, in any observationally viable model, quintessence becomes dynamically important only at low redshift, so it affords little purchase for measuring redshift dependence of its equation of state. Furthermore, as Figures 5-7 demonstrate, even a substantial transition in $n$ at low redshifts is very difficult to detect since the value of $n$ at $z=0$ is not known a priori, and time variation must therefore be judged relative to the constant- $n$ model that best mimics the time-variable model. Our broken power-law models have substantial low-redshift transitions by design, but there is usually a constant- $n$ model that predicts the same values of all observables to better than $1 \%$ at all observationally accessible redshifts. We conclude that detecting time variation of the equation of state requires truly extraordinary precision unless this variation occurs on a timescale much shorter than the Hubble time, which is possible but seems physically unlikely. Subpercent precision may be achievable by some methods (SN Ia observations look to us like the best hope), but it requires controlling systematic uncertainties, especially those that are correlated among different redshift bins, very tightly.

Our conclusions in this regard agree with those of Maor et al. (2001), who found that accurate measurements of the luminosity distance alone would be insufficient to determine the form of $w(z)$ for the dark matter energy component. Wang \& Garnavich (2001) and Tegmark (2001) showed that SN Ia measurements should be able to detect time variation in the energy density $\rho_{\phi}(z)$, but this only means demonstrating that $n>0(w>-1)$; we agree that a significant departure from $n=0$ should be detectable, but detecting time variation of $n$ is far more challenging. In a similar vein, despite fairly optimistic assumptions about the prospects for the SNAP satellite, Huterer \& Turner (2001) find that error bars on the time derivative of $w$ are quite large and degrade considerably with uncertainty in $\Omega_{m}$. Yamamoto et al. (2001) suggest that the form of the dark energy equation of state might be determined by studying strong gravitational lensing systems, but their results indicate that detecting time variation is possible only with extremely high precision measurements of the lensing systems and then only if $\Omega_{m, 0}$ is known precisely. The principal significance of our results, relative to these earlier papers, is that they apply to all proposed observable tests based on the cosmic expansion history since these tests always measure some combination of $H(z), d_{A}(z), t(z)$, or $D_{1}(z)$.

Our investigation shows that there is one generic form of time variation in the equation of state that might be observationally detectable. Constant $n$ models with $n \geq 2$ $\left(w \geq-\frac{1}{3}\right)$ are ruled out by current data, but a time-variable model could have $n \geq 2$ at high redshift and a transition to low $n$ at low redshift when quintessence becomes the dominant energy component. The Albrecht-Skordis model displays just this behavior since the quintessence roughly tracks the matter energy density $(n \approx 3)$ along the exponential part of $V(\phi)$ but changes its equation of state (to $w \approx-1, n \approx 0$ ) when it reaches the potential minimum. If $n \geq 2$ down to some fairly low redshift, then the dynamical effects of quintessence are nonnegligible (although small) over a fair fraction of the postrecombination expansion history, and they slow the progress of matter clustering. The result is a slight (few percent) mismatch between the value of $D_{1}$ at $z=z_{r} \approx 1100$ and the value expected for a constant- $n$ model that matches the low-redshift data; in observational terms, the level of CMB anisotropy would be a few percent higher than anticipated. Doran et al. (2001) emphasize a similar point and discuss the relation between $\mathrm{CMB}$ anisotropy and $\sigma_{8}$ in detail. Detecting even this type of time variation will be very challenging, requiring a precise determination of the effective low-redshift value of $n$, precise determinations of the present-day amplitude of matter clustering and $\Omega_{m, 0}$, and the demonstration that any excess CMB anisotropy does not arise from other sources, such as tensor fluctuations, secondary anisotropies, or contaminating foregrounds.

The discovery of dark energy is an extraordinary cosmological achievement, one that could happen only in the era of " precision cosmology." If the equation of state of this dark energy is substantially different from $p=-\rho$ or if it has been different in the recent past, then that departure should be detected independently by several of the ambitious observational efforts currently planned or underway. A precise $(\sim \pm 0.1)$ measurement of the low-redshift value of $w$ would be another extraordinary achievement, ruling out many models for the origin of dark energy and tightening the parameter space of others. However, the information provided by different observable probes of the cosmic expansion history, or by the same probe at different redshifts, is mostly redundant rather than complementary once $\Omega_{m, 0}$ has been determined to high precision. As a result, the next step of detecting time variation in the cosmic equation of state is likely to prove extremely difficult. If we are lucky, then the dark energy has the kind of dynamical significance at high redshift or sudden transition at low redshift that produces an observationally accessible signature, although reading that signature will still require a combination of several cosmological measurements of unprecedented precision. If we are not so fortunate, then the observable effects of the dark energy will, for the foreseeable future, provide only two numbers with which to describe it, the current energy density and an effective low-redshift value of $w$ (or some equivalent pair of parameters). Until a physical model comes along that accounts for these two numbers in a natural way without adjustable inputs, the true nature of the dark energy component is likely to remain mysterious.

A. M. L. and R. J. S. were supported in part by the DOE (DE FG02-91ER40690). D. H. W. was supported in part by the NSF (AST 00-98584). D. H. W. acknowledges the hospitality of the Institute for Advanced Study and financial support of the Ambrose Monell Foundation during the final phases of this work. We thank L. Amendola, R. Jimenez, E. Linder, and the anonymous referee for helpful comments on the manuscript.
Albrecht, A., \& Skordis, C. 2000, Phys. Rev. Lett., 84, 2076

Alcock, C., \& Paczynski, B. 1979, Nature, 281, 358 (AP)

Amendola, L. 2000, Phys. Rev. D, 62, 043511

Armendariz-Picon, C., Mukhanov, V., \& Steinhardt, P. J. 2000, Phys. Rev. Lett., 85,4438

\section{REFERENCES}

Astier, P. 2001, Phys. Lett. B, 500, 8

Baccigalupi, C. Balbi, A., Matarrese, S., Perrotta, F., \& Vittorio, N. 2002, Phys. Rev. D., 65, 063520

Bahcall, N. A., Fan, X., \& Cen, R. 1997, ApJ, 485, L53

Ballinger, W. E., Peacock, J. A., \& Heavens, A. F. 1996, MNRAS, 282, 877 
Barger, V., \& Marfatia, D. 2001, Phys. Lett. B, 498, 67

Benabed, K., \& Bernardeau, F. 2001, Phys. Rev. D, 64, 083501

Birkinshaw, M. 1999, Phys. Rep., 310, 97

Boyle, B. J., Shanks, T., Croom, S. M., Smith, R. J., Miller, L., Loaring, N., \& Heymans, C. 2000, MNRAS, 317, 1014

Boyle, L. A., Caldwell, R. R., \& Kamionkowski, M. 2001, preprint (astroph/0105318)

Caldwell, R. R., Dave, R., \& Steinhardt, P. J. 1998, Phys. Rev. Lett., 80, 1582

Calvão, M. O., de Mello Neto, J. R. T., \& Waga, I. 2002, Phys. Rev. Lett., 88,091302

Cappi, A. 2002, Astrophys. Lett. Commun., in press

Carlberg, R. G., Yee, H. K. C., \& Ellingson, E. 1997, ApJ, 478, 462

Chevallier, M., \& Polarski, D. 2001, Int. J. Mod. Phys. D, 10, 213

Chiba, T., \& Nakamura, T. 2000, Phys. Rev. D, 62, 121301

Cooray, A., Hu, W., Huterer, D., \& Joffre, M. 2001, ApJ, 557, L7

Cooray, A. R., \& Huterer, D. 1999, ApJ, 513, L95

Corasaniti, P. S., \& Copeland, E. J. 2002, Phys. Rev. D, 65, 043004

Croft, R. A. C., Weinberg, D. H., Bolte, M., Burles, S., Hernquist, L., Katz, N., Kirman, \& D., Tytler, D. 2001, ApJ, submitted (astro-ph/0012324)

Croft, R. A. C., Weinberg, D. H., Katz, N., \& Hernquist, L. 1998, ApJ, 495, 44

Croft, R. A. C., Weinberg, D. H., Pettini, M., Katz, N., \& Hernquist, L. 1999, ApJ, 520, 1

Dalal, N., Abazajian, K., Jenkins, E., \& Manohar, A. V. 2001, Phys. Rev. Lett., 87, 141302

Davé, R., Hernquist, L., Katz, N., \& Weinberg, D. H. 1999, ApJ, 511, 521

Doran, M., Lilley, M., \& Wetterich, C. 2002, Phys. Lett. B, 528, 175

Doran, M., Schwindt, J.-M., \& Wetterich, C. 2001, Phys. Rev. D, 64, 123520

Efstathiou, G. 1995, MNRAS, 274, L73

1999, MNRAS, 310,842

Eisenstein, D. J. 1997, preprint (astro-ph/9709054)

Eisenstein, D. J., et al. 2001, AJ, 122, 2267

Eke, V. R., Cole, S., \& Frenk, C. S. 1996, MNRAS, 282, 263

Ferreira, P. G., \& Joyce, M. 1997, Phys. Rev. Lett., 79, 4740

Freedman, W. L., et al. 2001, ApJ, 553, 47

Frenk, C. S., White, S. D. M., Efstathiou, G., \& Davis, M. 1990, ApJ, 351, 10

Garnavich, P. M., et al. 1998, ApJ, 509, 74

Gnedin, N. Y., \& Hamilton, A. J. S. 2001, MNRAS, submitted (astro-ph/ $0111194)$

Haiman, Z., Mohr, J. J., \& Holder, G. P. 2001, ApJ, 553, 545

Hamuy, M., Phillips, M. M., Suntzeff, N. B., Schommer, R. A., Maza, J., \& Aviles, R. 1996, AJ, 112, 2398

Heath, D. J. 1977, MNRAS, 179, 351

Hogg, D. W. 1999, preprint (astro-ph/9905116)

Hu, W. 2002, Phys. Rev. D, 65, 023003

Hui, L. 1999, ApJ, 519, L9

Hui, L., Stebbins, A., \& Burles, S. 1999, ApJ, 511, L5

Huterer, D. 2002, Phys. Rev. D, 65, 063001

Huterer, D., \& Turner, M. S. 2001, Phys. Rev. D, 64, 123527

Jimenez, R., \& Loeb, A. 2002, ApJ, 573, in preparation

Kolb, E. W., \& Turner, M. S. 1990, The Early Universe (Reading: Addison-Wesley)

Liddle, A. R., \& Scherrer, R. J. 1999, Phys. Rev. D, 59, 023509

Lima, J. A. S., \& Alcaniz, J. S. 2000, MNRAS, 317, 893 2002, ApJ, 566, 15

Mannheim, P. D. 2001, ApJ, 561, 1

Maoli, R., Van Waerbeke, L., Mellier, Y., Schneider, P., Jain, B.,

Bernardeau, F., Erben, T., \& Fort, B. 2001, A\&A, 368, 766

Maor, I., Brustein, R., \& Steinhardt, P. J. 2001, Phys. Rev. Lett., 86, 6

Martel, H., Shapiro, P. R., \& Weinberg, S. 1998, ApJ, 492, 29

Matsubara, T., \& Suto, Y. 1996, ApJ, 470, L1

Matsubara, T., \& Szalay, A. S. 2001, ApJ, 556, L67

McDonald, P. 2001, ApJ, submitted (astro-ph/0108064)

McDonald, P., \& Miralda-Escudé, J. 1999, ApJ, 518, 24
McDonald, P., Miralda-Escudé, J., Rauch, M., Sargent, W. L. W., Barlow, T. A., Cen, R., \& Ostriker, J. P. 2000, ApJ, 543, 1

Molnar, S. M., Birkinshaw, M., \& Mushotzky, R. F. 2002, ApJ, 570, 1

Nair, V. 1999, ApJ, 522, 569

Nakamura, T. T., Matsubara, T., \& Suto, Y. 1998, ApJ, 494, 13

Netterfield, C. B., et al. 2002, ApJ, 571, 604

Newman, J. A., \& Davis, M. 2000, ApJ, 534, L11 2002, ApJ, 564, 567

Newman, J. A., Marinoni, C., Coil, A. L., \& Davis, M. 2002, PASP, 114, 29

Ng, S. C. C., \& Wiltshire, D. L. 2001, Phys. Rev. D, 64, 123519

Outram, P. J., Hoyle, F., Shanks, T., Boyle, B. J., Croom, S. M., Loaring, N. S., Miller, L., \& Smith, R. J. 2001, MNRAS, 328, 174

Peacock, J. A., Jimenez, R., Dunlop, J. S., Waddington, I., Spinrad, H., Stern, D., Dey, A., \& Windhorst, R. A. 1998, MNRAS, 296, 1089

Peebles, P. J. E. 1980, The Large-Scale Structure of the Universe (Princeton: Princeton Univ. Press) Univ. Press)

Perlmutter, S., et al. 1999, ApJ, 517, 565

Phillipps, S. 1994, MNRAS, 269, 1077

Podariu, S., Nugent, P., \& Ratra, B. 2001, ApJ, 553, 39

Popowski, P. A., Weinberg, D. H., Ryden, B. S., \& Osmer, P. S. 1998, ApJ, 498, 11

Pryke, C., Halverson, N. W., Leitch, E. M., Kovac, J., Carlstrom, J. E., Holzapfel, W. L., \& Dragovan, M. 2002, ApJ, 568, 46

Ratra, B., \& Peebles, P. J. E. 1988, Phys. Rev. D, 37, 3406

Riess, A. G., et al. 1998, AJ, 116, 1009

Riess, A. G., Press, W. H., \& Kirshner, R. P. 1996, ApJ, 473, 88

Roukema, B. F., \& Mamon, G. A. 2000, A\&A, 358, 395

Ryden, B. S. 1995, ApJ, 452, 25

Sachs, R. K., \& Wolfe, A. M. 1967, ApJ, 147, 73

Saini, T. D., Raychaudhury, S., Sahni, V., \& Starobinsky, A. A. 2000, Phys. Rev. Lett., 85, 1162

Seljak, U. 1996, ApJ, 463, 1

2001, MNRAS, submitted (astro-ph/0111362)

Silveira, V., \& Waga, I. 1994, Phys. Rev. D, 50, 4890

Spergel, D., \& Pen, U. 1997, ApJ, 491, L67

Steinhardt, P. J., Wang, L., \& Zlatev, I. 1999, Phys. Rev. D, 59, 123504

Tegmark, M. 2001, preprint (astro-ph/0101354)

Turner, M. S., \& White, M. 1997, Phys. Rev. D, 56, 4439

Vandenberg, D. A., Stetson, P. B., \& Bolte, M. 1996, ARA\&A, 34, 461

van Dokkum, P. G., Franx, M., Kelson, D. D., \& Illingworth, G. D. 1998, ApJ, 504, L17

Vilenkin, A. 1985, Phys. Rep., 121, 263

Wang, L., Caldwell, R. R., Ostriker, J. P., \& Steinhardt, P. J. 2000, ApJ, 530,17

Wang, X., Tegmark, M., \& Zaldarriaga, M. 2001, Phys. Rev. D, submitted (astro-ph/0105091)

Wang, Y., \& Garnavich, P. M. 2001, ApJ, 552, 445

Wang, Y., \& Lovelace, G. 2001, ApJ, 562, L115

Weinberg, D. H., et al. 1999a, in Evolution of Large-Scale Structure: From Recombination to Garching, ed. A. J. Banday, R. K. Sheth, \& L. N. Da Costa (Enschede: ESO), 346

Weinberg, D. H., Croft, R. A. C., Hernquist, L., Katz, N., \& Pettini, M. 1999 b, ApJ, 522, 563

Weinberg, D. H., Hernquist, L., Katz, N., Croft, R., \& Miralda-Escude, J. 1997, in Proc. of the 13th IAP Colloq., Structure and Evolution of the IGM from QSO Absorption Line Systems, ed. P. Petitjean \& S. Charlot (Paris: Nouvelles Frontières), 133

Weller, J., \& Albrecht, A. 2001, Phys. Rev. Lett., 86, 1939

Weller, J., Battye, R., \& Kneissl, R. 2001, preprint (astro-ph/0110353)

Yamamoto, K., Kadoya, Y., Murata, T., \& Futamase, T. 2001, Prog. Theor. Phys., 106, 917

York, D. G., et al. 2000, AJ, 120, 1579

Zaldarriaga, M., Hui, L., \& Tegmark, M. 2001, ApJ, 557, 519

Zlatev, I., Wang, L., \& Steinhardt, P. J. 1999, Phys. Rev. Lett., 82, 896 